\title{
Generalized Single Integral Involving Multivariable Kampé De Fériet Function
}

\author{
Dashrath Singh Marko ${ }^{1}$, Sunil Pandey ${ }^{2}$, Manoj Kumar Shukla ${ }^{3}$ and \\ Rajeev Shrivastava ${ }^{4}$ \\ 1, 2,3 Department of Mathematics, Govt. Model Science College, Jabalpur, (M.P.), India \\ 4 Departments of Mathematics, Govt. Indira Gandhi Home Science Girls College, Shahdol, (M.P.), India
}

Abstract: The object of this paper is to obtain twenty five Eulerian type single integrals in the form of a general single integral involving multivariable extension of the Kampé de Fériet function [1]. The results are derived with the help of the generalized classical Watson's theorem obtained earlier by Lavoie et. Al. [2]. A few interesting special cases of our main result have also been discussed.

Key Words: Multivariable Kampé de Fériet function, Generalized Watson's theorem.

We make use of following abbreviation,

\section{Introduction}

$$
(\mathrm{a})_{\mathrm{k}}=\frac{\Gamma(\mathrm{a}+\mathrm{k})}{\Gamma(\mathrm{k})}=\mathrm{a}(\mathrm{a}+1) \ldots(\mathrm{a}+\mathrm{k}-1) ;
$$

and in what follows for the sake of brevity and elegance we recall the definition of multivariabe generalization of Kampé de Fériet function [1] in the notations of Burchnall and Chaundy [5]:

where

$$
\mathrm{F}_{\mathrm{l}: \mathrm{m}_{1} ; \cdots ; \mathrm{m}_{\mathrm{n}}}^{\mathrm{p}: \mathrm{q}_{1} ; \cdots ; \mathrm{q}_{\mathrm{n}}}\left[\begin{array}{l}
\left(\mathrm{a}_{\mathrm{p}}\right):\left(\mathrm{b}_{\mathrm{q}_{1}}^{\prime}\right) ; \cdots ;\left(\mathrm{b}_{\mathrm{q}_{\mathrm{n}}}^{(\mathrm{n})}\right) ; \\
\left(\alpha_{1}\right):\left(\beta_{\mathrm{m}_{1}}^{\prime}\right) ; \cdots ;\left(\beta_{\mathrm{m}_{\mathrm{n}}}^{(n)}\right) ;
\end{array} \mathrm{z}_{1} ; \cdots ; \mathrm{z}_{\mathrm{n}}\right]=\sum_{\mathrm{s}_{1} ; \cdots ; \mathrm{s}_{\mathrm{n}}=0}^{\infty} \Lambda\left(\mathrm{s}_{1} ; \cdots ; \mathrm{s}_{\mathrm{n}}\right) \frac{\mathrm{z}_{1} \mathrm{~s}_{1}}{\mathrm{~s}_{1} !} \cdots \frac{\mathrm{z}_{\mathrm{n}} \mathrm{s}_{\mathrm{n}}}{\mathrm{s}_{\mathrm{n}} !},
$$

and, for convergence of the multiple hypergeometric series in (1.1)

$$
\Lambda\left(s_{1} ; \cdots ; s_{n}\right)=\frac{\prod_{j=1}^{\mathrm{p}}\left(\mathrm{a}_{\mathrm{j}}\right)_{\mathrm{s}_{1}+\cdots+s_{n}} \prod_{j=1}^{\mathrm{q}_{1}}\left(\mathrm{~b}_{\mathrm{j}}^{\prime}\right)_{s_{1}} \cdots \prod_{j=1}^{\mathrm{q}_{\mathrm{n}}}\left(\mathrm{b}_{\mathrm{j}}^{(\mathrm{n})}\right)_{s_{n}}}{\prod_{\mathrm{j}=1}^{\mathrm{l}}\left(\alpha_{\mathrm{j}}\right)_{s_{1}+\cdots+s_{n}} \prod_{j=1}^{\mathrm{m}_{1}}\left(\beta_{\mathrm{j}}^{\prime}\right)_{s_{1}} \cdots \prod_{j=1}^{m_{n}}\left(\beta_{j}^{(n)}\right)_{s_{n}}}
$$

The equality holds when, in addition, either

$$
1+\mathrm{l}+\mathrm{m}_{\mathrm{k}}-\mathrm{p}-\mathrm{q}_{\mathrm{k}} \geq 0, \mathrm{k}=1,2, \cdots \mathrm{n} ;
$$

$$
\left|\mathrm{z}_{1}\right|^{1 /(\mathrm{p}-\mathrm{l})}+\cdots+\left|\mathrm{z}_{\mathrm{n}}\right|^{1 /(\mathrm{p}-\mathrm{l})}<1, \quad \text { if } \mathrm{p}>l
$$

or

$$
\max \left\{\left|\mathrm{z}_{1}\right|, \cdots\left|\mathrm{z}_{\mathrm{n}}\right|\right\}<1, \quad \text { if } \mathrm{p} \leq \mathrm{l} .
$$

Although the multiple hypergeometric series defined by (1.1) reduces to the Kampé de Fériet function [3] in the special case: $q_{1}=\cdots=q_{n}$ and $m_{1}=\cdots=m_{n}$.

The Kampé de Fériet function defined in (1.1) can be specialized to be expressed in terms of generalized hypergeometric series, among other things, as following instance:

$$
\begin{aligned}
& \mathrm{F}_{1: 0 ; \cdots ; 0}^{\mathrm{p}: 0 ; \cdots ; 0}\left[\begin{array}{l}
\alpha_{1}, \cdots, \alpha_{\mathrm{p}} \\
\beta_{1}, \cdots, \beta_{1} ; \cdots ; \mathrm{z}_{1} ;
\end{array}\right]={ }_{\mathrm{p}} \mathrm{F}_{1}\left[\begin{array}{l}
\alpha_{1}, \cdots, \alpha_{\mathrm{p}} \\
\beta_{1}, \cdots, \beta_{1}
\end{array} \mathrm{z}_{1}+\cdots+\mathrm{z}_{\mathrm{n}}\right] . \\
& \quad \mathrm{F}_{0: \mathrm{m}_{1} \cdots ; \mathrm{m}_{\mathrm{n}}}^{0: \mathrm{q}_{1} \cdots ; \mathrm{q}_{\mathrm{n}}}\left[\begin{array}{l}
-:\left(\mathrm{b}_{\mathrm{q}_{1}}^{\prime}\right) ; \cdots ;\left(\mathrm{b}_{\mathrm{q}_{\mathrm{n}}}^{(\mathrm{n})}\right) ; \\
-:\left(\beta_{\mathrm{m}_{1}}^{\prime}\right) ; \cdots ;\left(\beta_{\mathrm{m}_{\mathrm{n}}}^{(\mathrm{n})}\right) ; \mathrm{z}_{1} ; \cdots ; \mathrm{z}_{\mathrm{n}}
\end{array}\right] \\
& \quad={ }_{\mathrm{q}_{1}} \mathrm{~F}_{\mathrm{m}_{1}}\left[\begin{array}{l}
\mathrm{b}_{1}^{\prime}, \cdots, \mathrm{b}_{\mathrm{q}_{1}}^{\prime} ; \mathrm{z}_{1} \\
\beta_{1}^{\prime}, \cdots, \beta_{\mathrm{m}_{1}}^{\prime}, \cdots{ }_{\mathrm{q}_{\mathrm{n}}} \mathrm{F}_{\mathrm{m}_{\mathrm{n}}}\left[\begin{array}{l}
\mathrm{b}_{1}^{(\mathrm{n})}, \cdots, \mathrm{b}_{\mathrm{q}_{\mathrm{n}}}^{(\mathrm{n})} ; \mathrm{z}_{\mathrm{n}} \\
\beta_{1}^{(\mathrm{n})}, \cdots, \beta_{\mathrm{m}_{\mathrm{n}}}^{(\mathrm{n})}
\end{array}\right] .
\end{array}\right.
\end{aligned}
$$

For more details, see Karlsson [4, pp. 28-32].

\section{Results required}

The following results will be required in our present investigations.

$$
\int_{0}^{1} \mathrm{x}^{\mathrm{c}-1}(1-\mathrm{x})^{\mathrm{c}+\mathrm{j}-1}{ }_{2} \mathrm{~F}_{1}\left[\begin{array}{c}
\mathrm{a}, \mathrm{b} \\
\frac{1}{2}(\mathrm{a}+\mathrm{b}+\mathrm{i}+1)
\end{array} ; \mathrm{x}\right] \mathrm{dx}
$$




$$
=\frac{\Gamma(c) \Gamma(c+j)}{\Gamma(2 c+j)}{ }_{3} F_{2}\left[\begin{array}{c}
a, b, c \\
\frac{1}{2}(a+b+i+1), 2 c+j
\end{array} ; 1\right],
$$

provided $\Re(c)>0, \Re(c+j)>0$, and $\Re(2 c-a-b+i+1)>0$, for $i, j=0, \pm 1, \pm 2$. The result (2.1) is a special case of a general double integral given in Erdelyi et. Al. [6, pp. 399, Eq. (5)].

Lavoie et. Al. [2] have given the generalization of the Watson's theorem on the sum of a ${ }_{3} \mathrm{~F}_{2}$ and obtained the following twenty five results in the form of a single result:

$$
\begin{aligned}
& { }_{3} \mathrm{~F}_{2}\left[\begin{array}{c}
\mathrm{a}, \mathrm{b}, \mathrm{c} \\
\frac{1}{2}(\mathrm{a}+\mathrm{b}+\mathrm{i}+1), 2 \mathrm{c}+\mathrm{j}
\end{array} ; 1\right] \\
& =A_{i, j} \frac{2^{a+b+i-2} \Gamma\left(\frac{a+b+i+1}{2}\right) 2^{a+b+i-2} \Gamma\left(c+\left[\frac{j}{2}\right]+\frac{1}{2}\right) 2^{a+b+i-2} \Gamma\left(c-\frac{a+b+|i+j|-j-1}{2}\right)}{\Gamma\left(\frac{1}{2}\right) \Gamma(a) \Gamma(b)} \\
& \times\left\{\mathrm{B}_{\mathrm{i}, \mathrm{j}} \frac{\Gamma\left(\frac{\mathrm{a}}{2}+\frac{1-(-1)^{\mathrm{i}}}{4}\right) \Gamma\left(\frac{\mathrm{b}}{2}\right)}{\Gamma\left(\mathrm{c}-\frac{\mathrm{a}}{2}+\left[\frac{\mathrm{j}}{2}\right]+\frac{1}{2}-\frac{(-1)^{\mathrm{j}}\left(1-(-1)^{\mathrm{i}}\right)}{4}\right) \Gamma\left(\mathrm{c}-\frac{\mathrm{b}}{2}+\left[\frac{\mathrm{j}}{2}\right]+\frac{1}{2}\right)}\right. \\
& \left.+\mathrm{C}_{\mathrm{i}, \mathrm{j}} \frac{\Gamma\left(\frac{\mathrm{a}}{2}+\frac{1-(-1)^{\mathrm{i}}}{4}\right) \Gamma\left(\frac{\mathrm{b}}{2}+\frac{1}{2}\right)}{\Gamma\left(\mathrm{c}-\frac{\mathrm{a}}{2}+\left[\frac{\mathrm{j}+1}{2}\right]+\frac{(-1)^{\mathrm{j}}\left(1-(-1)^{\mathrm{i}}\right)}{4}\right) \Gamma\left(\mathrm{c}-\frac{\mathrm{b}}{2}+\left[\frac{\mathrm{j}+1}{2}\right]\right)}\right\},
\end{aligned}
$$

provided $\Re(2 c-a-b)>-1-i-2 j$, for $i, j=0, \pm 1, \pm 2$. Here, $[x]$ is the greatest integer less than or equal to $\mathrm{x}$ and $|\mathrm{x}|$ denotes the usual absolute value of $\mathrm{x}$. The coefficients $\mathrm{A}_{\mathrm{i}, \mathrm{j}}, \mathrm{B}_{\mathrm{i}, \mathrm{j}}$ and $\mathrm{C}_{\mathrm{i}, \mathrm{j}}$ are given respectively in [2].

\section{Main Results}

The following results for reducibility of multivariable Kampé de Fériet function will be established in this section.

$$
\begin{aligned}
& \begin{array}{c}
\int_{0}^{1} \mathrm{x}^{\mathrm{c}-1}(1-\mathrm{x})^{\mathrm{c}+\mathrm{j}-1}{ }_{2} \mathrm{~F}_{1}\left[\begin{array}{c}
\mathrm{a}, \mathrm{b} \\
\frac{1}{2}(\mathrm{a}+\mathrm{b}+\mathrm{i}+1)
\end{array} ; \mathrm{x}\right] \\
\mathrm{F}_{1: \sigma ; \cdots ; \sigma ; \rho}^{\mathrm{p}: \rho ; \cdots ;}\left[\begin{array}{l}
\left(\mathrm{a}_{\mathrm{p}}\right):\left(\mathrm{b}_{\rho}^{\prime}\right) ; \cdots ;\left(\mathrm{b}_{\rho}^{(\mathrm{n})}\right) ; \\
\left(\alpha_{1}\right):\left(\beta_{\sigma}^{\prime}\right) ; \cdots ;\left(\beta_{\sigma}^{(n)}\right) ;
\end{array} \mathrm{z}_{1} \mathrm{x}(1-\mathrm{x}) ; \cdots ; \mathrm{z}_{\mathrm{n}} \mathrm{x}(1-\mathrm{x})\right] \mathrm{dx}
\end{array} \\
& =\sum_{\mathrm{s}_{1} ; \cdots ; \mathrm{s}_{\mathrm{n}}=0}^{\infty} \Omega\left(\mathrm{s}_{1} ; \cdots ; \mathrm{s}_{\mathrm{n}}\right) \frac{\Gamma\left(\mathrm{c}+\sum_{\mathrm{i}=1}^{\mathrm{n}} \mathrm{s}_{\mathrm{i}}\right) \Gamma\left(\mathrm{c}+\mathrm{j}+\sum_{\mathrm{i}=1}^{\mathrm{n}} \mathrm{s}_{\mathrm{i}}\right)}{\Gamma\left(2 \mathrm{c}+\mathrm{j}+\sum_{\mathrm{i}=1}^{\mathrm{n}} 2 \mathrm{~s}_{\mathrm{i}}\right)} \\
& A_{i, j} \frac{2^{a+b+i-2} \Gamma\left(\frac{a+b+i+1}{2}\right) 2^{a+b+i-2} \Gamma\left(c+\sum_{i=1}^{n} s_{i}+\left[\frac{j}{2}\right]+\frac{1}{2}\right) 2^{a+b+i-2} \Gamma\left(c+\sum_{i=1}^{n} s_{i}-\frac{a+b+|i+j|-j-1}{2}\right)}{\Gamma\left(\frac{1}{2}\right) \Gamma(a) \Gamma(b)} \\
& \times\left\{\mathrm{B}_{\mathrm{i}, \mathrm{j}} \frac{\Gamma\left(\frac{\mathrm{a}}{2}+\frac{1-(-1)^{\mathrm{i}}}{4}\right) \Gamma\left(\frac{\mathrm{b}}{2}\right)}{\Gamma\left(\mathrm{c}+\sum_{\mathrm{i}=1}^{\mathrm{n}} \mathrm{s}_{\mathrm{i}}-\frac{\mathrm{a}}{2}+\left[\frac{\mathrm{j}}{2}\right]+\frac{1}{2}-\frac{(-1)^{\mathrm{j}}\left(1-(-1)^{\mathrm{i}}\right)}{4}\right) \Gamma\left(\mathrm{c}+\sum_{\mathrm{i}=1}^{\mathrm{n}} \mathrm{s}_{\mathrm{i}}-\frac{\mathrm{b}}{2}+\left[\frac{\mathrm{j}}{2}\right]+\frac{1}{2}\right)}\right. \\
& \left.+\mathrm{C}_{\mathrm{i}, \mathrm{j}} \frac{\Gamma\left(\frac{\mathrm{a}}{2}+\frac{1-(-1)^{\mathrm{i}}}{4}\right) \Gamma\left(\frac{\mathrm{b}}{2}+\frac{1}{2}\right)}{\Gamma\left(\mathrm{c}+\sum_{\mathrm{i}=1}^{\mathrm{n}} \mathrm{s}_{\mathrm{i}}-\frac{\mathrm{a}}{2}+\left[\frac{\mathrm{j}+1}{2}\right]+\frac{(-1)^{\mathrm{j}}\left(1-(-1)^{\mathrm{i}}\right)}{4}\right) \Gamma\left(\mathrm{c}+\sum_{\mathrm{i}=1}^{\mathrm{n}} \mathrm{s}_{\mathrm{i}}-\frac{\mathrm{b}}{2}+\left[\frac{\mathrm{j}+1}{2}\right]\right)}\right\},
\end{aligned}
$$

provided $\Re(c)>0$, for $j=-1,-2 ; \Re(c+j)>0$ for $j=0,1,2$. Also,

(i) $1+\mathrm{l}+\sigma-\mathrm{p}-\rho \geq 0$,

(ii) $\left|\mathrm{z}_{1}\right|^{1 /(\mathrm{p}-\mathrm{l})}+\cdots+\left|\mathrm{z}_{\mathrm{n}}\right|^{1 /(\mathrm{p}-\mathrm{l})}<1$, if $\mathrm{p}>l$ and $\max \left\{\left|\mathrm{z}_{1}\right|, \cdots\left|\mathrm{z}_{\mathrm{n}}\right|\right\}<1$, if $\mathrm{p} \leq \mathrm{l}$.

Also, the coefficients $A_{i, j}, B_{i, j}$ and $C_{i, j}$ can be obtain easily from the tables given in [2] by replacing $c$ by $\mathrm{c}+\mathrm{s}_{1}+\cdots+\mathrm{s}_{\mathrm{n}}$, and $\Omega\left(\mathrm{s}_{1} ; \cdots ; \mathrm{s}_{\mathrm{n}}\right)$ is defined as 


$$
\Omega\left(s_{1} ; \cdots ; s_{n}\right)=\frac{\prod_{j=1}^{p}\left(a_{j}\right)_{s_{1}+\cdots+s_{n}} \prod_{j=1}^{\rho}\left(b_{j}^{\prime}\right)_{s_{1}} \cdots \prod_{j=1}^{\rho}\left(b_{j}^{(n)}\right)_{s_{n}}}{\prod_{j=1}^{l}\left(\alpha_{j}\right)_{s_{1}+\cdots+s_{n}} \prod_{j=1}^{\sigma}\left(\beta_{j}^{\prime}\right)_{s_{1}} \cdots \prod_{j=1}^{\sigma}\left(\beta_{j}^{(n)}\right)_{s_{n}}} .
$$

\section{Proof of (3.1)}

To prove (3.1), we proceed as follows : Let

$$
\begin{aligned}
I= & \int_{0}^{1} x^{c-1}(1-x)^{c+j-1}{ }_{2} F_{1}\left[\begin{array}{c}
a, b \\
\frac{1}{2}(a+b+i+1)
\end{array} ; x\right] \\
& F_{1: \sigma ; \cdots ; \cdots ; \sigma}^{p: \cdots ; \rho}\left[\begin{array}{c}
\left(a_{p}\right):\left(b_{\rho}^{\prime}\right) ; \cdots ;\left(b_{\rho}^{(n)}\right) ; \\
\left(\alpha_{1}\right):\left(\beta_{\sigma}^{\prime}\right) ; \cdots ;\left(\beta_{\sigma}^{(n)}\right) ;
\end{array} z_{1} x(1-x) ; \cdots ; z_{n} x(1-x)\right] d x .
\end{aligned}
$$

Expressing multivariable Kampé de Fériet function in series form as defined in (1.1), we have

$$
\begin{aligned}
& I=\int_{0}^{1} x^{c-1}(1-x)^{c+j-1}{ }_{2} F_{1}\left[\begin{array}{c}
a, b \\
\frac{1}{2}(a+b+i+1)
\end{array} ; x\right] \\
& \sum_{s_{1} ; \cdots ; s_{n}=0}^{\infty} \Omega\left(s_{1} ; \cdots ; s_{n}\right)[x(1-x)]^{s_{1}} \cdots[x(1-x)]^{s_{1}} \frac{z_{1}^{s_{1}}}{s_{1} !} \cdots \frac{z_{n}^{s_{n}}}{s_{n} !} d x,
\end{aligned}
$$

where $\Omega\left(\mathrm{s}_{1} ; \cdots ; \mathrm{s}_{\mathrm{n}}\right)$ is given with (3.2).

Changing the order of integration and summation which is justified due to uniformly convergence of the series, we obtain

$$
\begin{gathered}
I=\sum_{s_{1} ; \cdots ; s_{n}=0}^{\infty} \Omega\left(s_{1} ; \cdots ; s_{n}\right) \frac{z_{1} s_{1}}{s_{1} !} \cdots \frac{z_{n}^{s_{n}}}{s_{n} !} \\
\times\left\{\int_{0}^{1} x^{c+\sum_{i=1}^{n} s_{i}-1}(1-x)^{c+\sum_{i=1}^{n} s_{i}+j-1}{ }_{2} F_{1}\left[\begin{array}{c}
a, b \\
\frac{1}{2}(a+b+i+1)
\end{array} ; x\right] d x\right\},
\end{gathered}
$$

which, upon using (2.1), becomes

$$
\begin{gathered}
I=\sum_{s_{1} ; \cdots ; s_{n}=0}^{\infty} \Omega\left(s_{1} ; \cdots ; s_{n}\right) \frac{z_{1}^{s_{1}}}{s_{1} !} \cdots \frac{z_{n}{ }^{s_{n}}}{s_{n} !} \frac{\Gamma\left(c+\sum_{i=1}^{n} s_{i}\right) \Gamma\left(c+j+\sum_{i=1}^{n} s_{i}\right)}{\Gamma\left(2 c+j+\sum_{i=1}^{n} 2 s_{i}\right)} \\
\times{ }_{3} F_{2}\left[\begin{array}{c}
a, b, \quad c+\sum_{i=1}^{n} s_{i} \\
\frac{1}{2}(a+b+i+1), 2 c+\sum_{i=1}^{n} 2 s_{i}+j
\end{array} ; 1\right] .
\end{gathered}
$$

By making use of (2.2) and replacing $\mathrm{c}$ by $\mathrm{c}+\mathrm{s}_{1}+\cdots+\mathrm{s}_{\mathrm{n}}$, we finally arrive at the right-hand side of (3.1). This completes the proof of (3.1).

\section{Special cases}

In this section, we shall mention some of the interesting special cases of our main result (3.1).

(i) If we take $\mathrm{i}=\mathrm{j}=0$ in (3.1), then we have, after a little simplification, the following transformation formula:

$$
\begin{aligned}
& \int_{0}^{1} \mathrm{x}^{\mathrm{c}-1}(1-\mathrm{x})^{\mathrm{c}-1}{ }_{2} \mathrm{~F}_{1}\left[\begin{array}{c}
\mathrm{a}, \mathrm{b} \\
\frac{1}{2}(\mathrm{a}+\mathrm{b}+1)
\end{array} ; \mathrm{x}\right] \\
& \mathrm{F}_{1: \sigma ; \cdots ; \sigma}^{\mathrm{p}: \rho ; \cdots ; \rho}\left[\begin{array}{l}
\left(\mathrm{a}_{\mathrm{p}}\right):\left(\mathrm{b}_{\rho}^{\prime}\right) ; \cdots ;\left(\mathrm{b}_{\rho}^{(\mathrm{n})}\right) ; \\
\left(\mathrm{\alpha}_{1}\right):\left(\beta_{\sigma}^{\prime}\right) ; \cdots ;\left(\beta_{\sigma}^{(\mathrm{n})}\right) ;
\end{array} \mathrm{z}_{1} \mathrm{x}(1-\mathrm{x}) ; \cdots ; \mathrm{z}_{\mathrm{n}} \mathrm{x}(1-\mathrm{x})\right] \mathrm{dx} \\
& =\frac{2^{\mathrm{a}+\mathrm{b}+2 \mathrm{c}-1} \Gamma\left(\frac{\mathrm{a}+\mathrm{b}+1}{2}\right) \Gamma\left(\frac{\mathrm{a}}{2}\right) \Gamma\left(\frac{\mathrm{b}}{2}\right) \Gamma(\mathrm{c}) \Gamma\left(\mathrm{c}-\frac{\mathrm{a}}{2}-\frac{\mathrm{b}}{2}+\frac{1}{2}\right)}{\Gamma(\mathrm{a}) \Gamma(\mathrm{b}) \Gamma\left(\mathrm{c}-\frac{\mathrm{a}}{2}+\frac{1}{2}\right) \Gamma\left(\mathrm{c}-\frac{\mathrm{b}}{2}+\frac{1}{2}\right)} \\
& \times \mathrm{F}_{1+2: \sigma ; \cdots ; \sigma ; \rho}^{\mathrm{p}+2: \cdots ; \rho}\left[\begin{array}{c}
\left(\mathrm{a}_{\mathrm{p}}\right), \mathrm{c}, \mathrm{c}+\frac{1-\mathrm{a}-\mathrm{b}}{2}:\left(\mathrm{b}_{\rho}^{\prime}\right) ; \cdots ;\left(\mathrm{b}_{\rho}^{(\mathrm{n})}\right) ; \\
\left(\alpha_{1}\right), \mathrm{c}+\frac{1-\mathrm{a}}{2}, \mathrm{c}+\frac{1-\mathrm{b}}{2}:\left(\beta_{\sigma}^{\prime}\right) ; \cdots ;\left(\beta_{\sigma}^{(\mathrm{n})}\right) ; \frac{\mathrm{z}_{1}}{4} ; \cdots ; \frac{\mathrm{z}_{\mathrm{n}}}{4}
\end{array}\right],
\end{aligned}
$$

provided that the conditions easily obtainable from (3.1) are satisfied. 
(ii) In (3.1), if we take $\mathrm{i}=0 ; \mathrm{j}=-1$ then we have, after a little simplification, the following transformation formula:

$$
\begin{aligned}
& \int_{0}^{1} \mathrm{x}^{\mathrm{c}-1}(1-\mathrm{x})^{\mathrm{c}-2}{ }_{2} \mathrm{~F}_{1}\left[\begin{array}{c}
\mathrm{a}, \mathrm{b} \\
\frac{1}{2}(\mathrm{a}+\mathrm{b}+1) ; \mathrm{x}
\end{array}\right] \\
& \mathrm{F}_{1: \sigma ; \cdots ; \sigma}^{\mathrm{p}: \rho ; \cdots ; \rho}\left[\begin{array}{l}
\left(\mathrm{a}_{\mathrm{p}}\right):\left(\mathrm{b}_{\rho}^{\prime}\right) ; \cdots ;\left(\mathrm{b}_{\rho}^{(\mathrm{n})}\right) ; \\
\left(\mathrm{a}_{\mathrm{l}}\right):\left(\beta_{\sigma}^{\prime}\right) ; \cdots ;\left(\beta_{\sigma}^{(\mathrm{n})}\right) ;
\end{array} \mathrm{z}_{1} \mathrm{x}(1-\mathrm{x}) ; \cdots ; \mathrm{z}_{\mathrm{n}} \mathrm{x}(1-\mathrm{x})\right] \mathrm{dx} \\
& =\frac{2^{\mathrm{a}+\mathrm{b}+2 \mathrm{c}} \Gamma\left(\frac{\mathrm{a}+\mathrm{b}+1}{2}\right) \Gamma\left(\frac{\mathrm{a}}{2}\right) \Gamma\left(\frac{\mathrm{b}}{2}\right) \Gamma(\mathrm{c}-1) \Gamma\left(\mathrm{c}-\frac{\mathrm{a}}{2}-\frac{\mathrm{b}}{2}-\frac{1}{2}\right)}{\Gamma(\mathrm{a}) \Gamma(\mathrm{b}) \Gamma\left(\mathrm{c}-\frac{\mathrm{a}}{2}+\frac{1}{2}\right) \Gamma\left(\mathrm{c}-\frac{\mathrm{b}}{2}-\frac{1}{2}\right)}
\end{aligned}
$$

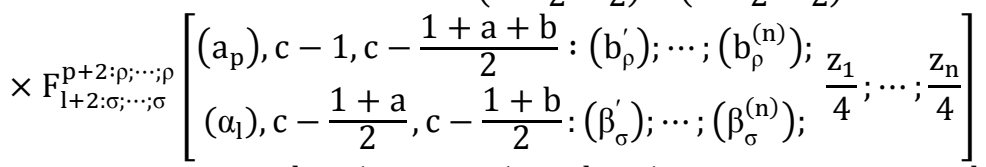

$$
\begin{aligned}
& +\frac{2^{\mathrm{a}+\mathrm{b}-2 \mathrm{c}} \Gamma\left(\frac{\mathrm{a}+\mathrm{b}+1}{2}\right) \Gamma\left(\frac{\mathrm{a}}{2}+\frac{1}{2}\right) \Gamma\left(\frac{\mathrm{b}}{2}+\frac{1}{2}\right) \Gamma(\mathrm{c}-1) \Gamma\left(\mathrm{c}-\frac{\mathrm{a}}{2}-\frac{\mathrm{b}}{2}-\frac{1}{2}\right)}{\Gamma(\mathrm{a}) \Gamma(\mathrm{b}) \Gamma\left(\mathrm{c}-\frac{\mathrm{a}}{2}\right) \Gamma\left(\mathrm{c}-\frac{\mathrm{b}}{2}\right)} \\
& \times \mathrm{F}_{1+2: \sigma ; \cdots ; \sigma}^{\mathrm{p}+2: \rho ; \rho ;}\left[\begin{array}{c}
\left(\mathrm{a}_{\mathrm{p}}\right), \mathrm{c}-1, \mathrm{c}-\frac{1+\mathrm{a}+\mathrm{b}}{2}:\left(\mathrm{b}_{\rho}^{\prime}\right) ; \cdots ;\left(\mathrm{b}_{\rho}^{(\mathrm{n})}\right) ; \mathrm{z}_{1} \\
\left(\alpha_{\mathrm{l}}\right), \mathrm{c}-\frac{\mathrm{a}}{2}, \mathrm{c}-\frac{\mathrm{b}}{2}:\left(\beta_{\sigma}^{\prime}\right) ; \cdots ;\left(\beta_{\sigma}^{(\mathrm{n})}\right) ;
\end{array}\right],
\end{aligned}
$$

provided that the conditions easily obtainable from (3.1) are satisfied.

(iii) If we take $\mathrm{i}=0 ; \mathrm{j}=1$ in (3.1), then we have, after a little simplification, the following transformation formula:

$$
\begin{aligned}
& \int_{0}^{1} x^{c-1}(1-x)^{c-2}{ }_{2} F_{1}\left[\begin{array}{c}
a, b \\
\frac{1}{2}(a+b+1)
\end{array} ; x\right] \\
& F_{l: \sigma ; \cdots ; \sigma}^{p: \rho ; \cdots ; \rho}\left[\begin{array}{l}
\left(a_{p}\right):\left(b_{\rho}^{\prime}\right) ; \cdots ;\left(b_{\rho}^{(n)}\right) ; \\
\left(\alpha_{l}\right):\left(\beta_{\sigma}^{\prime}\right) ; \cdots ;\left(\beta_{\sigma}^{(n)}\right) ;
\end{array} z_{1} x(1-x) ; \cdots ; z_{n} x(1-x)\right] d x \\
& =\frac{2^{a+b-2 c-2} \Gamma\left(\frac{a+b+1}{2}\right) \Gamma\left(\frac{a}{2}\right) \Gamma\left(\frac{b}{2}\right) \Gamma(c-1) \Gamma\left(c-\frac{a}{2}-\frac{b}{2}+\frac{1}{2}\right)}{\Gamma(a) \Gamma(b) \Gamma\left(c-\frac{a}{2}+\frac{1}{2}\right) \Gamma\left(c-\frac{b}{2}+\frac{1}{2}\right)} \\
& \times F_{l+2: \sigma ; \cdots ; \sigma}^{p+2: \rho ; \rho}\left[\begin{array}{c}
\left(a_{p}\right), c, c+\frac{1-a-b}{2}:\left(b_{\rho}^{\prime}\right) ; \cdots ;\left(b_{\rho}^{(n)}\right) ; \\
\left(\alpha_{l}\right), c+\frac{1-a}{2}, c+\frac{1-b}{2}:\left(\beta_{\sigma}^{\prime}\right) ; \cdots ;\left(\beta_{\sigma}^{(n)}\right) ;
\end{array} ;\right. \\
& \left.-\frac{2^{a+b-2 c-2} \Gamma\left(\frac{a+b+1}{2}\right) \Gamma\left(\frac{a}{2}+\frac{1}{2}\right) \Gamma\left(\frac{b}{2}+\frac{1}{2}\right) \Gamma(c) \Gamma\left(c-\frac{a}{2}-\frac{b}{2}-\frac{1}{2}\right)}{(a+1}\right) \Gamma(c-b+1) \\
& \Gamma(a) \Gamma(b) \Gamma\left(c-\frac{a+1}{2}\right) \Gamma\left(c-\frac{b}{2}+1\right)
\end{aligned}
$$

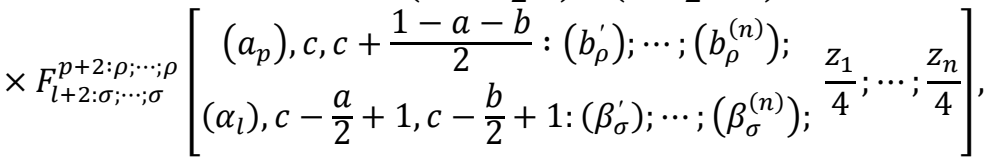

provided that the conditions easily obtainable from (3.1) are satisfied. Similarly other results can also be obtained.

\section{References}

[1] Srivastava, H.M. and Panda, R.: Some analytic and asymptotic confluent expansions for functions of several variables. Math. Comput. 29 (1975), 1115-1128.

[2] Lavoie, J.L. et. Al.: Generalizations of Watson's theorem on the sum of a ${ }_{3} \mathrm{~F}_{2}$, Indian J. Math. 32, (1992), no. 1, $23-32$.

[3] Appell, P. et. Kampé de Fériet, J.: Fonctions Hypergeometriques et Hyper-spheriques Polynomes d'Hermite, Gauthier-Villars, Paris, (1926), p. 150 (Eq. 29).

[4] Karlsson, P.W.: Reduction of certain generalized Kampé de Fériet functions, Math. Scand. 32, (1973), $265-268$.

\section{Books :}

[5] Burchnall, J.L. and Chaundy, T.Y.: Expansions of Appell's double hypergeometric functions, Quart. J. Math., Oxford Series, 12, (1941), 112-128.

[6] Erdelyi, A. et. Al.: Tables of integral transforms, Vol. II, McGraw-Hill, New York, (1954). 